\title{
Cystic neurohypophysial Germinoma associated with Moyamoya disease
}

\author{
Yasushi Shibata, M.D., Ph.D., Masahide Matsuda, M.D., \\ Kensuke Suzuki, M.D., Ph.D., Akira Matsumura, M.D., Ph.D., \\ Department of Neurosurgery, Institute of Clinical Medicine, \\ Graduate School of Comprehensive Human Sciences, University of Tsukuba, \\ Tsukuba, Ibaraki, 305-0005, Japan
}

Address for correspondence

Yasushi Shibata, M.D., Ph.D.

Department of Neurosurgery, University of Tsukuba,

Tennodai 1-1-1 Tsukuba, Ibaraki, 305-0005, Japan

Tel: +81-298-53-3220

Fax: +81-298-53-3214

E-mail: yshibata@md.tsukuba.ac.jp 


\section{Abstract}

Moyamoya disease associated with a germinoma has never yet been reported in the literature.

This report describes a case of a cystic neurohypophysial germinoma associated with

moyamoya disease. A fifteen-year-old girl with central diabetes insipidus was admitted to the hospital. A radiological examination revealed a suprasellar tumor and moyamoya disease.

Surgery and a pathological study confirmed a pure germinoma. After the patient was treated with radiation and chemotherapy, the tumor was controlled without any effect on the moyamoya vessels. This report describes a rare incidental combination of moyamoya disease and a suprasellar germinoma. In the management of such cases, physicians should be aware of the symptoms, course, and natural history of both diseases and the effects and side effects of each type of therapy.

\section{Key words}

brain tumor; cystic tumor; germinoma; moyamoya disease; suprasellar tumor 


\section{Introduction}

Moyamoya disease is an intracranial vascular disease characterized by stenosis of intracranial arteries and "moyamoya vessels”. There are some reports of moyamoya disease associated with brain tumors. However, no cases of moyamoya disease associated with a germinoma have ever been reported in the literature. This report describes a patient with a cystic neurohypophysial germinoma and moyamoya disease. The relationship between the two diseases and the management of the patient with these 2 different pathologies is discussed.

\section{Case report}

The patient was 15-year-old girl. She has experienced thirst and polyuria since November of 2005. She had experienced amenorrhea since February 2006. Occasional headaches occurred, beginning in June 2006. She was referred to the hospital, because head computed tomography (CT) showed a suprasellar mass lesion. She had no remarkable medical or family history. She had no previous episodes of cerebral ischemia. Her right visual acuity was slightly decreased.

No visual field defect was observed. There were no other neurological abnormalities. An endocrine examination revealed complete central diabetes insipidus and a low base line value of growth hormone and gonadotropin. Her menstrual cycle was irregular. The human chorionic gonadotropin (HCG)- $\beta$ levels in her blood and cerebrospinal fluid were slightly elevated. Other tumor markers were normal.

Brain Magnetic resonance imaging (MRI) revealed a suprasellar cystic tumor of 
iso-intensity on T1 and T2 weighted images. The parenchymal part of tumor was homogeneously enhanced with gadolinium-diethylene triamine pentaacetic acid (Gd-DTPA, Figure 1: coronal image). The tumor involved the optic nerve and pituitary grand. MRI and magnetic resonance angiography (MRA) showed a hypoplastic left internal carotid artery and left middle cerebral artery (MCA; not shown). Cerebral angiography confirmed the nearly complete occlusion of the internal carotid artery at the supraclinoid portion and left moyamoya vessels (Figure 2: A-P view). Neither tumor staining nor the compression of the cerebral vessels by the suprasellar tumor was observed. Technetium-99m ethyl cysteinate dimer single photon emission CT (ECD SPECT) at rest showed mild hypoperfusion in the left MCA area. After the intravenous injection of diamox, a sufficient increase of bilateral cerebral blood flow was recognized.

Tumor biopsy surgery was performed via a right pterional craniotomy to avoid potential damage to left moyamoya vessels. The right superficial temporal artery was preserved for future bypass surgery. During surgery, the PaCO2 was kept slightly high to maintain sufficient cerebral blood flow. The tumor margin was not recognized under the surgical microscope. Swelling was recognized in the right optic nerve and chiasma. A cystic mass lesion was seen behind the chiasma and yellowish cyst fluid and tumor tissue in the cystic mass were removed for a pathological examination.

Pathologically, the tumor showed 2 cell patterns typical of a germinoma (Figure 3: 
Hematoxylin and Eosin staining, original magnification 400X). Immunostaining was positive staining for placental alkaline phosphatase (PLAP), and very low for HCG- $\beta$. There was no evidence of syncytiotrophblastic giant cells (STGC), so the pathological diagnosis was a pure germinoma. The Ki-67 labeling index was higher than 60\%.

The patient received 2 cycles of chemotherapy consisting of carboplatin and etoposide. MRI after the 2 cycles of the chemotherapy showed a remarkable decrease of tumor. The patient received focal and whole ventricle fractionated conventional radiation therapy, with a total dose of $40 \mathrm{~Gy}$. She underwent a third cycle of the same chemotherapy after the radiation. Then tumor disappeared on MRI. Tumor recurrence has not been seen at 2 years after the onset. The radiation and chemotherapy did not affect the cerebral blood flow. MRA and ECD SPECT showed same findings after the radiation and chemotherapy as in the initial studies. No symptoms associated with moyamoya disease have yet been observed.

\section{Discussion}

There are four possible mechanisms to explain a combination of a brain tumor and moyamoya disease (9). These are 1) Direct compression of arteries by the tumor, 2) secondary effects of radiation to the tumor, 3) combination with neurocutaneous syndrome, 4) incidental combination. In the present case, the moyamoya vessels were seen only in the left MCA area. There was no direct compression of the cerebral artery by tumor. Therefore, this case appears to represent an incidental combination. 
A literature search revealed 10 cases of incidental combination of a brain tumor and moyamoya disease $(1-6,8,12,13)$ (Table 1$)$. The ages of patients ranged from 3 to 66 , the median age was 31.5 year-old. Most patient were children or young adults. There is no gender difference. There were 3 astrocytomas, 3 meningiomas, 3 pituitary adenomas and 1 craniopharyngioma. Moyamoya diseases were found during the investigation of the brain tumors in all of those patients. In some patients, the moyamoya disease became symptomatic after the therapy for the brain tumor. Some patients underwent revascularization surgery.

The optimal treatment strategy for patients with a dual pathology remains to be elucidated. In the present case no symptoms associated with moyamoya disease have so far been observed. Radiation therapy might damage the intracranial artery and affect cerebral blood flow. We believe, however, the radiation therapy with an appropriate dose and field could not be avoided, because a germinoma is a lethal malignant tumor. Without appropriate radiation and chemotherapy tumor recurrence is inevitable and such tumor recurrence clearly threatens both the quality of life and survival itself $(7,10)$. As a result, even though there is some risk of alterations in the cerebral blood flow due to a progression of the moyamoya disease in response to radiation therapy, since radiation should be administered to control the germinoma cells. Both the cerebral blood flow and possible tumor recurrence will be closely annually followed in this patient using ECD SPECT, Gd-DTPA enhanced MRI and MRA. Revascularization surgery would be considered if ischemic symptoms caused by moyamoya 
disease are later arise.

Although cystic suprasellar (neurohypophysial) germinomas are very rare, one case has been previously reported (11). Cystic changes may cause rapid compression of the optic nerve or other parasellar structures. Complete surgical removal of the suprasellar tumor is not easy because there are many critical structures involved, such as the optic nerve, pituitary stalk and hypothalamus. The cyst itself was a well recognized good target of potential surgery. Surgical opening of the thin cyst wall is a less invasive procedure in comparison to the removal of a solid tumor. Cyst opening is also effective to decrease the tumor mass and limit the radiation field. Therefore, surgical cyst opening and tumor biopsy are a reasonable surgical strategy for treating a cystic suprasellar tumor.

In conclusion this report described a rare incidental combination of moyamoya disease and a suprasellar germinoma. In the management of such cases, the physician should be aware of the symptoms, course and natural history of both diseases and the effects and side effects of each therapy. 


\section{Legend of figures}

Figure 1: Gd-DTPA enhanced coronal T1 weighted MRI shows an enhanced suprasellar cystic tumor.

Figure 2: A-P view of the left internal carotid arteriogram shows stenosis of the carotid artery terminal and moyamoya vessels.

Figure 3: Hematoxylin and eosin staining of the pathological specimen of the suprasellar tumor shows a mixture of large round tumor cells and small dense nuclear lymphocytes, the so-called two-cell pattern. (original magnification 400x)

\section{References}

1. Aihara N, Nagai H, Mase M, Kanai H, Wakabayashi S, Mabe H:(1992) Atypical Moyamoya disease associated with brain tumor. Surg Neurol 37:46-50

2. Arita K, Uozumi T, Oki S, Kuwabara S, Ohba S, Nakahara T, et al:(1992) Moyamoya disease associated with pituitary adenoma--report of two cases. Neurol Med Chir (Tokyo) 32:753-757

3. Hattori M, Tokunaga J, Toya S, Shizawa H:(1973) A occulusion of the circle of Willis after the removal of cerebellar astrocytoma. No to Hattatsu(Jpn with English astract) 5:319-314

4. Kaku M, Nakayama T, Matsukado Y:(1973) [Surgical case of meninigioma associated with basal abnormal vascular network: author translation]. Shujyutsu [Japanese] 27:1159-1162

5. Kitano S, Sakamoto H, Fujitani K, Kobayashi Y:(2000) Moyamoya disease associated with a brain stem glioma. Childs Nerv Syst 16:251-255

6. Lau YL, Milligan DW:(1986) Atypical presentation of craniopharyngioma associated with Moyamoya disease. J R Soc Med 79:236-237

7. Matsutani M:(2001) Combined chemotherapy and radiation therapy for CNS germ cell tumors--the Japanese experience. J Neurooncol 54:311-316. 
8. Mizukawa N, Iwatsuki K:(1973) [Case of cerebral basal rete mirable associated with brain tumor]. Rinsho Shinkeigaku(Jpn with English astract) 13:650-657

9. Mori K, Takeuchi J, Ishikawa M, Handa H, Toyama M, Yamaki T:(1978) Occlusive arteriopathy and brain tumor. J Neurosurg 49:22-35

10. Osuka S, Tsuboi K, Takano S, Ishikawa E, Matsushita A, Tokuuye K, et al:(2007) Long-term outcome of patients with intracranial germinoma. J Neurooncol 83:71-79

11. Suzuki K, Sonobe M, Matsutani M, Sugita K, Takahashi S:(1999) Suprasellar cystic germinoma. Childs Nerv Syst 15:134-136

12. Tada T, Ishii K, Oshima S, Hara H, Kobayashi S:(1992) Secretory meningioma associated with numerous meningothelial rosettes. Acta Neuropathol 84:342-345

13. Uchida K, Arakawa Y, Ohyama K, Sirakawa M, Tsuji R, Yokoyama M, et al:(2003) Growth hormone-secreting pituitary adenoma associated with primary moyamoya disease--case report. Neurol Med Chir (Tokyo) 43:356-359 
Figure 1

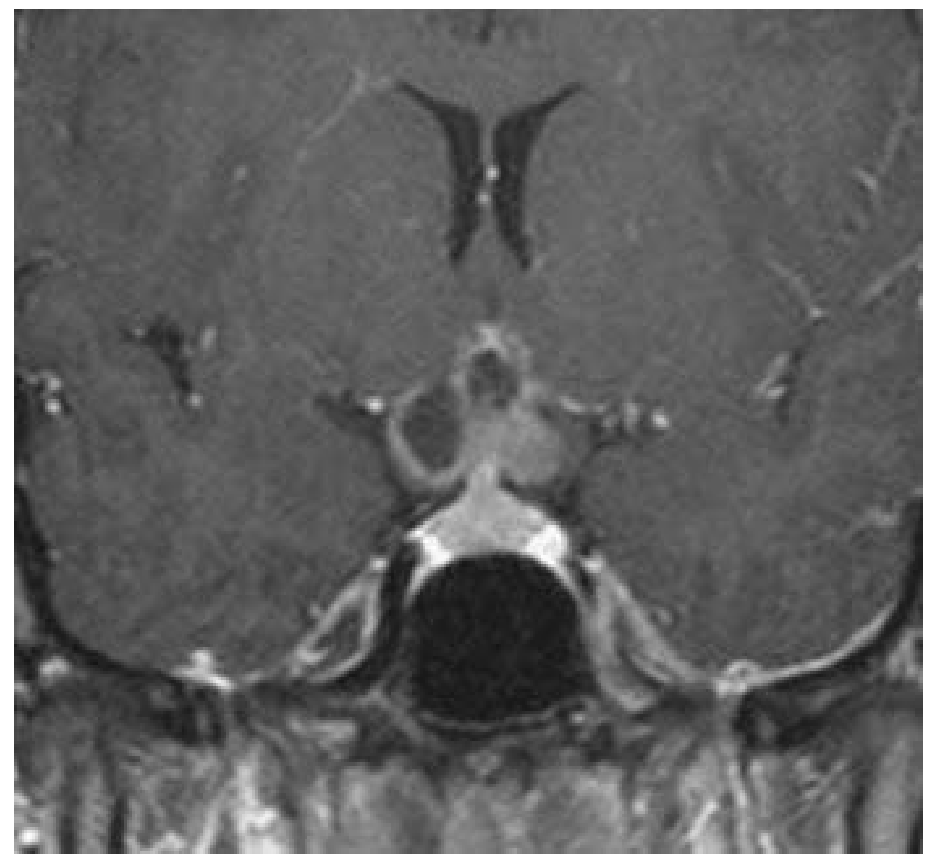


Figure 2

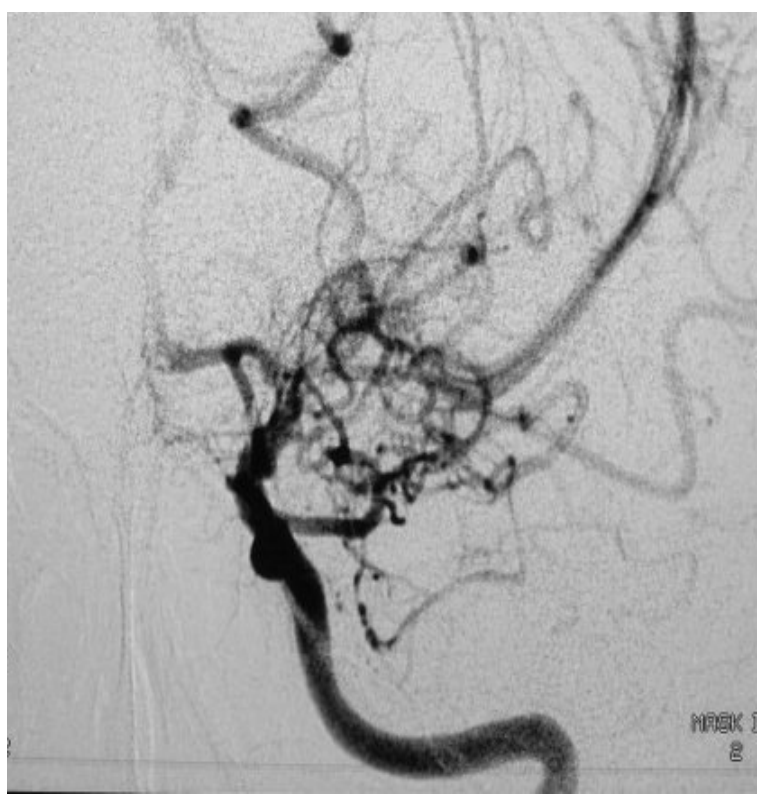


Figure 3

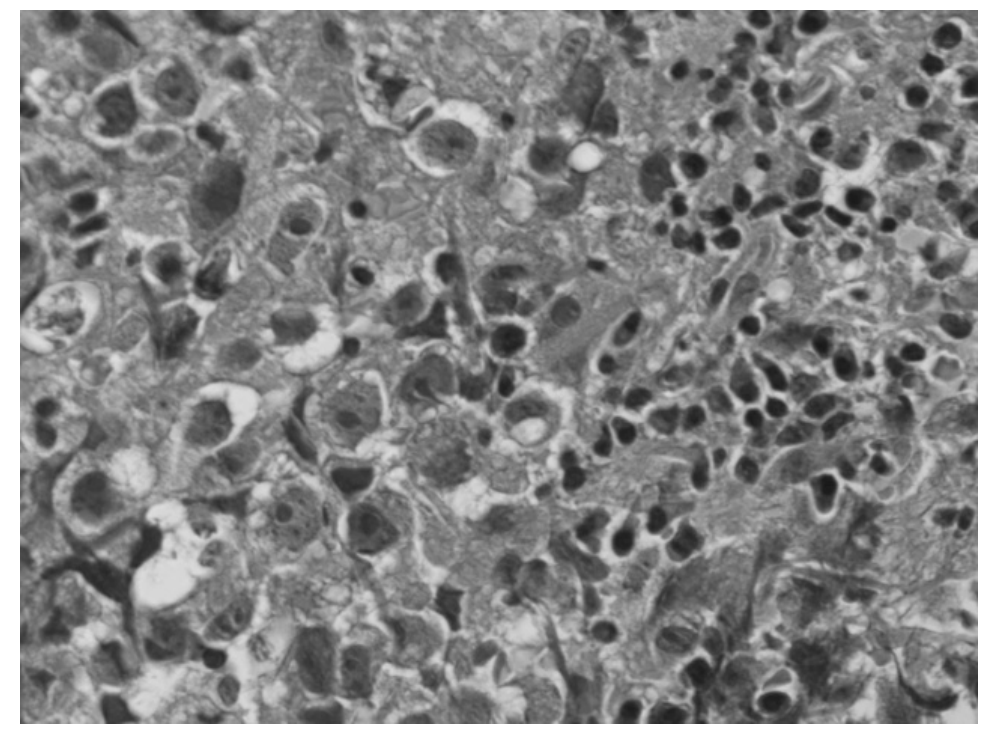


Table. 1

Reported cases of incidental combination of a brain tumor and moyamoya disease

\begin{tabular}{cccl}
\hline Case & Age & Sex & pathology and location of brain tumor \\
\hline 1 & 4 & M & Pilocytic astrocytoma at of cerebellar vermis \\
2 & 40 & F & PRL-producing pituitary adenoma \\
3 & 23 & F & PRL-producing pituitary adenoma \\
4 & 3 & M & Craniopharyngioma \\
5 & 40 & F & GH-producing adenoma \\
6 & 44 & F & Malignant meningioma at temporo-occipital \\
7 & 40 & F & Meningioma at sphenoid ridge \\
8 & 66 & M & Secretory meningioma at left convexity \\
9 & 8 & M & Pilocytic astrocytoma at brain stem \\
10 & 11 & M & Astrocytoma at cerebellum \\
11 & 15 & F & Germinoma at suprasellar (our case)
\end{tabular}

